\title{
GMZ-1 is a podophyllotoxin derivative that suppresses growth and induces apoptosis in adriamycin-resistant K562/A02 cells through modulation of MDR1 expression
}

\author{
BO CAO ${ }^{1,2^{*}}$, SHUWANG YANG ${ }^{1 *}$, WUWEI LI ${ }^{3}$, HONG CHEN ${ }^{2}$, YAZE CHEN $^{1}$, YONGFENG LIU $^{1,4}$ and BIN LIU ${ }^{1}$ \\ ${ }^{1}$ Department of Pharmacognosy, Logistics University of Chinese People's Armed Police Forces, Tianjin 300309; \\ ${ }^{2}$ Tianjin Key Laboratory of Cardiovascular Remodeling and Target Organ Injury, Tianjin 300162; ${ }^{3}$ Department of \\ Pharmacology, School of Basic Medicine, Tianjin Medical University, Tianjin 300070; ${ }^{4}$ Armed Police Corps Inner \\ Mongolia Unit Hospital, Urumqi, Hohhot, Inner Mongolia 010040, P.R. China
}

Received October 19, 2016; Accepted August 8, 2017

DOI: $10.3892 / \mathrm{mmr} .2017 .7862$

\begin{abstract}
The incidence of multidrug resistance (MDR) during cancer chemotherapy is a major challenge for treatment. With the aim of identifying drugs that are capable of targeting treatment-resistant cancer cells, the present study evaluated the efficacy of GMZ-1 in cancer chemotherapy using K562/A02, an MDR leukemia cell line. Cell viability and apoptosis were measured by MTT assay and flow cytometry/Giemsa staining, respectively. The expression levels of the MDR protein 1 (MDR1) gene transcript and protein in K562/A02 cells were determined by reverse transcription-quantitative polymerase chain reaction and western blot analyses, respectively. GMZ-1 suppressed the viability of various human cancer cell lines and induced apoptosis in the K562/A02 cell line in a time- and concentration-dependent manner. GMZ-1 toxicity may be associated with a decrease in MDR gene expression. These findings demonstrated that GMZ-1 may have efficacy as a potential antitumor drug to overcome leukemia cell resistance to apoptosis induced by chemotherapy.
\end{abstract}

\section{Introduction}

The majority of patients with cancer respond to initial chemotherapy (1); however, many patients subsequently relapse following this initial response. These patients are commonly characterized by the emergence of drug-resistant cells and

Correspondence to: Dr Yongfeng Liu or Dr Bin Liu, Department of Pharmacognosy, Logistics University of Chinese People's Armed Police Forces, 1 Huizhihuan Road, Tianjin 300309, P.R. China

E-mail:694991184@qq.com

E-mail: iamicehe@163.com

*Contributed equally

Key words: GMZ-1, K562/A02, multidrug resistance, VP-16, multidrug resistance protein 1 consequent resistance to multiple anti-cancer agents, which may have various chemical structures and mechanisms of action (2). This phenomenon, defined as multidrug resistance (MDR), is a major cause of chemotherapy failure. There are several potential mechanisms of resistance; one involves elevated expression of membrane transporter proteins and, therefore, declined intracellular drug concentrations and cytotoxicity. Among these transporter proteins, MDR protein 1 (MDR1), encoded by the MDR1 gene, has been associated with the resistance phenotype (3).

Various inhibitors of the drug efflux pump, including calcium channel blockers, anti-arrhythmics, antidepressants and antipsychotics, have been demonstrated to overcome drug resistance in vitro $(4,5)$. However, a number of these were demonstrated to exhibit high toxicity in animal studies (6). Others that belong to the class of MDR modulators or chemosensitizers are less cytotoxic and are able to reverse MDR1-associated resistance (7).

Podophyllotoxin is an interesting lead in the development of anticancer antiviral agents. Toxicity issues and side effects cause its limited use. Etoposide (VP-16) and teniposide (VM-26), derivatives of podophyllotoxin, have been successfully used in combination chemotherapy. Cancers like small cell lung cancer, testicular cancer, acute leukaemia and malignant lymphoma responded to them well. However, these derivatives have not overcome limitations, such as narrow anticancer spectrum, low solvability and development of resistance. In addition, major side effects including gastroenteric reaction and leukopenia have restricted their usage. The present study designed and filtered a series of water soluble derivatives of podophyllotoxin. To the best of our knowledge, there has been no report on the role of podophyllotoxin or its analogues in MDR reversal, particularly in MDR leukemia K562/A02 cells. Therefore, a number of novel podophyllotoxin derivatives were synthesized and their cytotoxicity in K563/A02 cells was tested. The present study proposed that the novel derivative GMZ-1 may be an alternative to VP-16, a clinical anti-cancer agent (Fig. 1). In order to investigate this, the anti-proliferative capacity of GMZ-1 was assayed in a number of cancer cell lines; as GMZ-1 exhibited high 


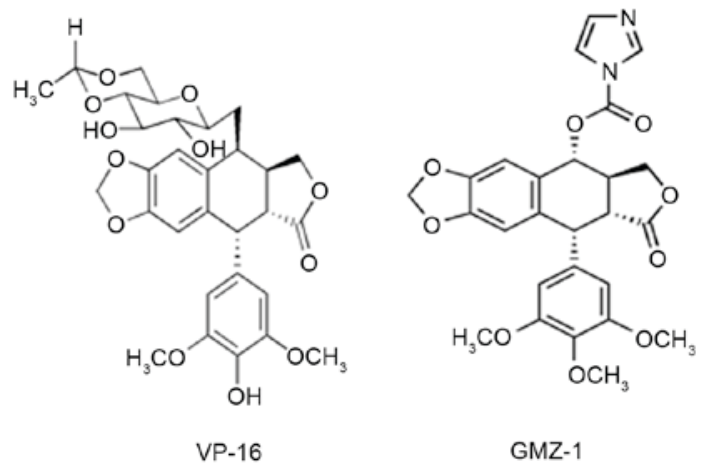

Figure 1. Chemical structures of VP-16 and GMZ-1.

toxicity towards K562, an MDR cell line, these cells were subsequently used to compare the effects of GMZ-1 and VP-16 in vitro. It was observed that GMZ-1 inhibited proliferation and induced apoptosis in K562/A02 cells in a time- and concentration-dependent manner. The present study additionally investigated the underlying mechanism of the anticancer activity of GMZ.

\section{Materials and methods}

Cell lines and culture. The K562 cell line (courtesy of Professor Hong Chen, Logistics University of Chinese People's Armed Police Forces, Tianjin, China) was a clone from human chronic myelogenous leukemia, previously established by alternate passages in nude mice and in vitro culture. HeLa, A549, MCF-7, HepG2, SKOV3, BGC-823, MGC-803 and the fibroblast cell line (3T3) were purchased from Type Culture Collection of the Chinese Academy of Sciences (Shanghai, China). K562 was cultured in RPMI-1640 (catalog no. 31800-022) supplemented with $10 \%$ fetal bovine serum (catalog no. 10099141) (both from Gibco, Thermo Fisher Scientific, Inc., Waltham, MA, USA) at $37^{\circ} \mathrm{C}$ in a humidified $5 \% \mathrm{CO}_{2}$ atmosphere. The MDR leukemia cell line K562/A02 (courtesy of Professor Hong Chen also) was generated previously by incremental adriamycin (ADM) treatments refer to Yang's et al paper published in 1995 (1). K562/A02 was maintained in RPMI-1640 medium supplemented with $1 \mu \mathrm{g} / \mathrm{ml}$ ADM to maintain its MDR phenotype.

Cell viability measurement. In order to evaluate the anti-proliferative activity of GMZ-1 [molecular weight 508.15, white powder, insoluble in water, purity $>98 \%$, supplied by Professor Hong Chen (Tianjin Key Laboratory of Cardiovascular Remodeling and Target Organ Injury, Tianjin, China). GMZ-1 was synthesized with imidazole-2-carboxy to generate podophyllolox imidazole-2-carboxylate] in cancer cell lines, cell viability was measured by determining mitochondrial dehydrogenase activity using an MTT assay. Cells $\left(5 \times 10^{3}\right.$ cells/well) were plated in 96-well plates and cultured overnight. Triplicate wells were treated with concentrations of GMZ-1 (10, 1, 0.1 and $0.01 \mu \mathrm{mol} / \mathrm{l})$ and VP-16 (10, 1, 0.1 and $0.01 \mu \mathrm{mol} / \mathrm{l}$ ) (cat no. H32025583; prepared with normal saline; Jiangsu Hengrui Medicine Co., Ltd., Lianyungang, China) or normal saline (control vehicle) for $48 \mathrm{~h}$. To perform the MTT assay, $20 \mu \mathrm{l}$ MTT solution $(5 \mathrm{mg} / \mathrm{ml}$ in PBS; Sigma-Aldrich;
Merck KGaA, Darmstadt, Germany) was added to each well, followed by incubation for $4 \mathrm{~h}$ at $37^{\circ} \mathrm{C}$. A total of $150 \mu \mathrm{l} /$ well dimethyl sulfoxide was added at room temperature for $10 \mathrm{~min}$ to dissolve the formazan precipitate. Absorbance was measured at a wavelength of $570 \mathrm{~nm}$ (Thermo Fisher Scientific, Inc.).

Flow cytometry analysis. K562/A02 cells ( $5 \times 10^{3}$ cells/well) were seeded in 6-well plates and cultured overnight. Triplicate wells were treated with $0.05,0.10$ or $0.20 \mu \mathrm{M}$ GMZ- 1 and $10 \mu \mathrm{M}$ VP-16 or normal saline (vehicle control) for 12, 24 and 36 h. Cells were collected and fixed in $70 \%$ ethyl alcohol at $4^{\circ} \mathrm{C}$ overnight, followed by washing in PBS and incubation with $10 \mu \mathrm{g} / \mathrm{ml} \mathrm{RNA}$ se at $37^{\circ} \mathrm{C}$ for $30 \mathrm{~min}$. Cells were subsequently incubated with $10 \mu \mathrm{g} / \mathrm{ml}$ propidium iodide (PI) for $30 \mathrm{~min}$ in the dark on ice. The stained samples were analysed using a FACSCalibur (BD Biosciences, Franklin Lakes, NJ, USA). Data was analysed using FlowJo version 7.6.3 software (FlowJo LLC, Ashland, OR, USA).

Giemsa staining. K562/A02 cells were treated with varying concentrations of GMZ-1 or normal saline (control vehicle) for $48 \mathrm{~h}$, lifted from the plate and mounted on slides. Following rinsing with water, the slides were stained with Giemsa solution (BDH; Merck $\mathrm{KGaA}$ ) for $5 \mathrm{~min}$ at room temperature. The slides were rinsed with water three times and the cells were observed under an inverted microscope (TMS; Nikon Corporation, Tokyo, Japan) at x400 magnification.

Examination of MDR1 gene expression by reverse transcription-quantitative polymerase chain reaction ( $R T$-qPCR). RNA was isolated from K562 or K562/A02 cells using TRIzol reagent (Invitrogen; Thermo Fisher Scientific, Inc.), and RT-qPCR was performed using a SuperScript One-Step RT-PCR kit (Invitrogen; Thermo Fisher Scientific, Inc.) in a $50 \mu 1$ reaction mix. The reaction mix contained $25 \mu 12 \mathrm{X}$ RT-PCR buffer, $3 \mu$ l template RNA, $0.6 \mu$ l forward and reverse primers each ( $\beta$-actin $540 \mathrm{bp}$, sense, 5'-GTGGGGCGCCCC AGGCACCA-3' and antisense, 5'-CTTCCTTAATGTCAC GCACGATTTC-3'; MDR-1, 150 bp, sense, 5'-GTGGGGCGC CCCAGGCACCA-3' and antisense, 5'-CTTCCTTAATGT CACGCACGATTTC-3'), $1 \mu \mathrm{l}$ AMV/Taq mixture and $19.8 \mu \mathrm{l}$ deionised water. The thermocycling reaction protocol was as follows: Reverse transcription for $35 \mathrm{~min}$ at $37^{\circ} \mathrm{C}$; pre-denaturation at $94^{\circ} \mathrm{C}$ for $3 \mathrm{~min} ; 30$ cycles of qPCR ( 1 min denaturation at $94^{\circ} \mathrm{C}, 30 \mathrm{sec}$ annealing at $57^{\circ} \mathrm{C}$ and $1 \mathrm{~min}$ extension at $72^{\circ} \mathrm{C}$ ); and $10 \mathrm{~min}$ final extension at $72^{\circ} \mathrm{C}$. PCR products were run on $1.5 \%$ agarose gels with $0.01 \%$ Gel Red (cat no. G5560; Beijing Solarbio Science and Technology Co., Ltd., Beijing, China), with $\beta$-actin (540 bp) as an internal standard. Band intensity was quantified using Gel-Pro Analyser 3.1 (Media Cybernetics, Inc., Rockville, MD, USA).

Examination of MDR1 protein expression by western blot analysis. Cells were lysed using rdioimmunoprecipitation acid lysis buffer (cat no. P0013B; Beyotime Institute of Biotechnology, Haimen, China) and the extracted protein was quantified with a bicinchoninic protein assay kit (cat no. P0010; Beyotime Institute of Biotechnology). A total of $30 \mu \mathrm{g} /$ well cell extracts were separated by Bolt ${ }^{\mathrm{TM}} 12 \%$ Bis-Tris Plus 10-well gels (cat no. NW00120BOX; Thermo Fisher Scientific, 
Table I. Cytotoxic activity of GMZ-1 on human cancer cells and fibroblasts.

\begin{tabular}{lcr}
\hline & \multicolumn{2}{c}{$\mathrm{IC}_{50}(\mu \mathrm{M})$} \\
\cline { 2 - 3 } Cell line & \multicolumn{1}{c}{ GMZ-1 } & \multicolumn{1}{c}{ VP-16 } \\
\hline HeLa & $0.07 \pm 0.01$ & $1.33 \pm 0.86$ \\
A549 & $0.18 \pm 0.07$ & $1.06 \pm 0.73$ \\
MCF-7 & $0.14 \pm 0.05$ & $2.36 \pm 0.53$ \\
HepG-2 & $0.093 \pm 0.012$ & $2.03 \pm 0.55$ \\
SKOV3 & $0.12 \pm 0.04$ & $3.43 \pm 0.87$ \\
BGC-823 & $0.083 \pm 0.009$ & $2.06 \pm 0.59$ \\
MGC-803 & $0.089 \pm 0.011$ & $3.61 \pm 0.85$ \\
3T3 & $0.34 \pm 0.07$ & $17.36 \pm 2.29$ \\
\hline
\end{tabular}

Values are presented as the mean \pm standard deviation $(n=3) . \mathrm{IC}_{50}$, half-maximal inhibitory concentration; VP-16, etoposide.

Table II. Reversion of drug resistance in K562/A02 cells .

\begin{tabular}{lcrc}
\hline & \multicolumn{3}{c}{$\mathrm{IC}_{50}(\mu \mathrm{M})$} \\
\cline { 2 - 4 } Drug & $\mathrm{K} 562$ & $\mathrm{~K} 562 / \mathrm{A} 02$ & Fold change \\
\hline Adriamycin & $0.26 \pm 0.10$ & $28.62 \pm 4.27$ & 110.08 \\
VP-16 & $2.02 \pm 0.83$ & $22.81 \pm 4.23$ & 11.29 \\
GMZ-1 & $0.08 \pm 0.02$ & $0.12 \pm 0.03$ & 1.52 \\
\hline
\end{tabular}

Values are presented as the mean \pm standard deviation $(n=3) . \mathrm{IC}_{50}$, half-maximal inhibitory concentration; VP-16, etoposide.

Inc.), and transferred to nitrocellulose membranes (Bio-Rad Laboratories, Inc., Hercules, CA, USA). The membranes were blocked with 5\% non-fat milk in PBS containing $0.1 \%$ Tween- 20 for $1 \mathrm{~h}$ at room temperature and incubated overnight at $4^{\circ} \mathrm{C}$ with MDR1/ABCB1 (E1Y7B) rabbit monoclonal antibody (cat no. 13342; Cell Signaling Technology, Inc., Danvers, MA, USA) at 1:1,000 dilution or GAPDH (D16H11) XP ${ }^{\circledR}$ rabbit monoclonal antibody (cat no. 5174; Cell Signaling Technology, Inc.) at 1:1,000 dilution. Following washing, the membranes were incubated with a horse radish peroxidase-conjugated secondary antibody, anti-rabbit IgG, horse radish peroxidase-conjugated antibody (cat no. 7074; Cell Signaling Technology, Inc.) at 1:3,000 dilution at room temperature for $1 \mathrm{~h}$ and visualized using SuperSignal ${ }^{\mathrm{TM}}$ West Pico Plus Chemiluminescent substrate (cat no. 34580; Thermo Fisher Scientific, Inc.).

Statistics. All data are presented as the mean \pm standard error. Differences between groups were analysed using a one-way analysis of variance followed by Student-Newman-Keuls and Least Significant Difference post hoc tests using SPSS version 20 software (IBM Corp., Armonk, NY, USA). $\mathrm{P} \leq 0.05$ was considered to indicate a statistically significant difference.

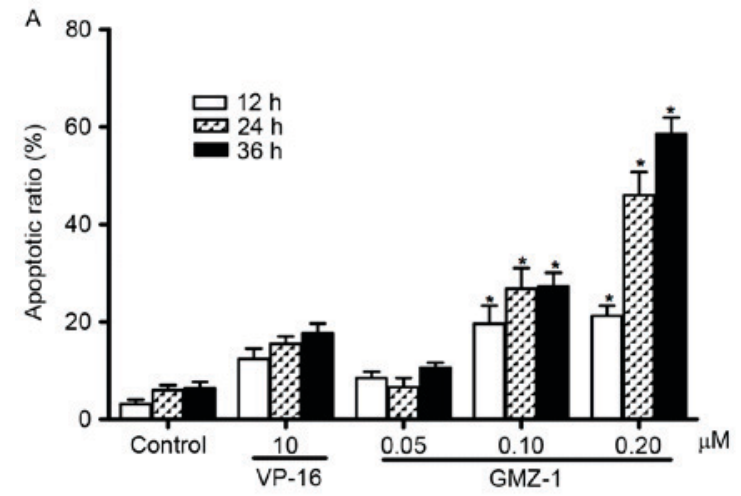

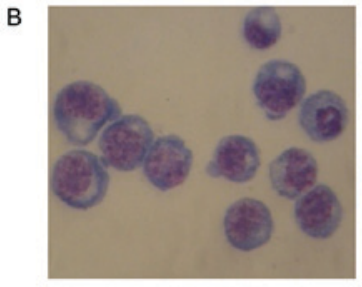

Control

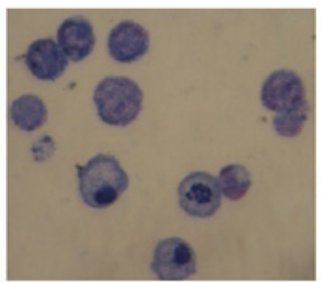

$\mathrm{GMZ}-10.10 \mu \mathrm{M}$

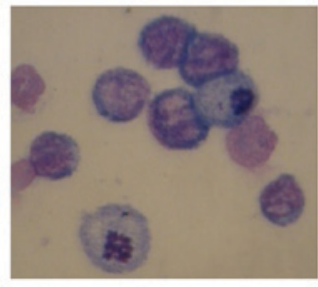

GMZ-1 $0.05 \mu \mathrm{M}$

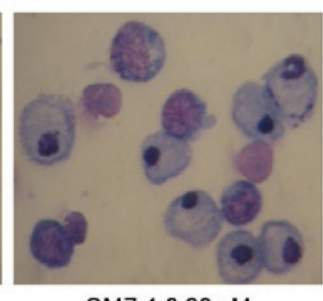

$\mathrm{GMZ}-10.20 \mu \mathrm{M}$
Figure 2. GMZ-1 induces apoptosis in K562/A02 cell line. (A) Flow cytometric analysis of K562/A02 cells treated with GMZ-1 or VP-16 for 12-36 h. Apoptosis was assessed by propidium iodide staining and flow cytometry. ${ }^{*} \mathrm{P}<0.05$ vs. respective control. (B) Morphology of K562/A02 cells treated with increasing concentrations of GMZ-1. K562/A02 cells were treated with vehicle or different concentrations of GMZ-1 for $48 \mathrm{~h}$ and stained with Giemsa. Nuclear condensation, cytoplasmic shrinkage and the formation of apoptotic bodies were visible in GMZ-1 treated cells. Representative images of three independent experiments are presented. Magnification, x400.

\section{Results}

GMZ-1 reduces cancer cell viability. GMZ-1 demonstrated a marked effect on the viability of several cancer cell lines, and the half-maximal inhibitory concentration $\left(\mathrm{IC}_{50}\right)$ values following treatment for $48 \mathrm{~h}$ are presented in Table I. GMZ-1 displayed the highest efficacy in K562 and K562/A02 cells, with $\mathrm{IC}_{50}$ values of $0.08 \pm 0.02$ and $0.12 \pm 0.03 \mu \mathrm{M} 48 \mathrm{~h}$ following treatment, respectively (Table II). Therefore, the K562/A02 cell line was selected as a model to examine the impact of GMZ-1 on cell viability.

GMZ-1 induces apoptosis in K562/A02 cells. A number of anti-cancer drugs impact upon apoptosis-associated signaling pathways to induce apoptosis in cancer cells. In order to examine whether the reduced viability of K562/A02 cells was due to the induction of apoptosis, flow cytometry analysis of PI-stained cells was performed. K562/A02 cells were treated with $0.05,0.10$ or $0.20 \mu \mathrm{M} \mathrm{GMZ}-1$ for 12,24 or $36 \mathrm{~h}$. The flow cytometry results indicated that GMZ-1 may induce apoptosis in K562/A02 cells in a time- and concentration-dependent manner (Fig. 2A). Quantification revealed a significant 
A

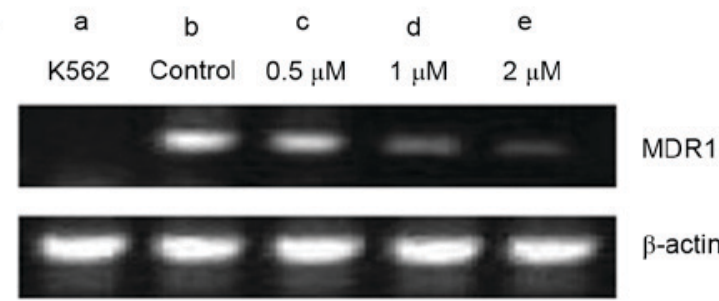

B

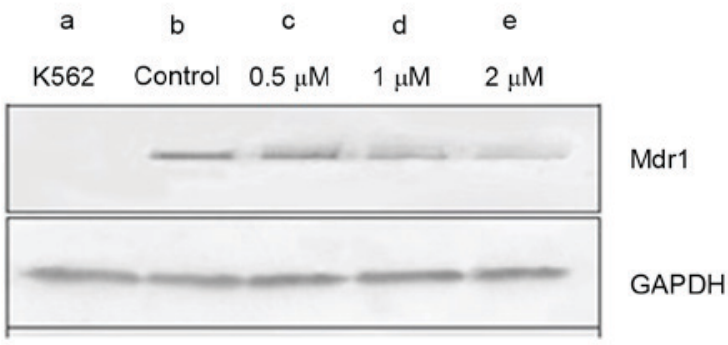

Figure 3. Quantification of MDR1 expression in GMZ-1-treated K562 and K562/A02 cells. (A) The level of MDR1 mRNA in K562 and K562/A02 treated with various concentrations of GMZ-1 was determined using reverse transcription-quantitative polymerase chain reaction analysis. The expression of MDR1 mRNA in (a) K562 cells and K562/A02 cells treated with (b) $0.1 \%$ DMSO, (c) $0.05 \mu \mathrm{M}$ GMZ-1, (d) $0.1 \mu \mathrm{M}$ GMZ-1 or (e) $0.2 \mu \mathrm{M}$ GMZ-1 is presented. $\beta$-actin mRNA served as the loading control. (B) Expression of MDR1 protein in K562 and K562/A02 cells treated with various concentrations of GMZ-1, determined by western blotting. The expression of MDR1 protein in (a) K562 cells and K562/A02 cells treated with (b) $0.1 \%$ DMSO, (c) $0.05 \mu \mathrm{M}$ GMZ-1, (d) $0.1 \mu \mathrm{M}$ GMZ-1 or (e) $0.2 \mu \mathrm{M}$ GMZ-1 is presented GAPDH served as the loading control. MDR1, multidrug resistance protein 1; DMSO, dimethyl sulfoxide.

difference in the apoptotic rate between control cells and cells treated with GMZ-1 (P<0.05; Fig. 2A).

GMZ-1 treated K562/A02 cells were stained with Giemsa solution to observe whether GMZ-1 induced the characteristic morphology of apoptosis. The observed morphology in GMZ-1-treated K562/A02 cells included nuclear condensation, cytoplasmic shrinkage and the formation of apoptotic bodies (Fig. 2B), which were absent in the control cells. Consistent with the MTT assay, these results indicated that GMZ-1 may reduce the viability of K562/A02 cells via the induction of apoptosis.

Expression analysis of the MDR1 gene and MDR1 protein in K562 and K562/A02 cells. The MDR1 gene, encoding MDR1, is associated with MDR. The present study examined MDR1 expression at the mRNA and protein levels in K562 and K562/A02 cells, using RT-qPCR and western blot analyses. Cells were treated with various concentrations of GMZ-1 $(0.05,0.1$ and $0.2 \mu \mathrm{M})$ for $24 \mathrm{~h}$. A marked increase in MDRI gene and MDR1 protein expression was observed in K562/A02 cells, compared with K562 cells (Fig. 3). Notably, MDR1 and MDR1 expression decreased with an increasing concentration of GMZ-1, which suggested that K562 was an MDR1-negative cell line and K562/A02 was an MDR1-overexpressing cell line.

\section{Discussion}

Although novel chemotherapeutic drugs are being developed, chemotherapy remains a challenge in cancer treatment. This is partially due to the development of MDR. Among the numerous mechanisms underlying MDR, elevated expression of the MDRl-encoded MDR1 protein in cancer cells has been considered to be a frequent factor $(8,9)$. MDR1 serves to remove the drug from the cells, thereby assisting in drug resistance. The weak potency and toxicity of developed MDR modulators have limited their clinical use. The few non-toxic compounds that downregulate the expression of MDR1 include curcumin (10), tryptanthrin (11), estrogen (12) and perospirone (13).

VP-16, an aryltetralinelignan, is a clinical antitumor drug used to treat testicular cancer and small cell lung cancer $(14,15)$. VP-16 elicits a few adverse effects, including myelosuppression and the initiation of secondary leukaemia (14,16-18). In order to reduce damage to bone marrow cells, VP-16 has been combined with other compounds in animal studies, such as quercetin, dexrazoxane and wongonin (19-21).

The present study demonstrated that the novel podophyllotoxin derivative GMZ-1 exhibited increased efficacy compared with a traditional podophyllotoxin derivative (22). GMZ-1 has a similar $\mathrm{IC}_{50}$ value between MDR1-negative cell line K562 and overexpressing cell line K562/A02; however, it exhibits decreased cytotoxicity in human fibroblasts at therapeutic doses. Apoptosis is characterized by specific morphological changes including plasma membrane blebbing, chromatin condensation and fragmentation, and the emergence of apoptotic bodies. The results of the present study suggested that GMZ-1 may induce apoptosis in K562/A02 in vitro and significantly decrease MDR1 expression from $24 \mathrm{~h}$. To the best of our knowledge, this is the first report demonstrating the suppressive effect of GMZ-1 on MDR1 expression in K562/A02 cells.

In conclusion, GMZ-1, as a novel derivative of podophyllotoxin, may have utility as an MDR modulator in adriamycin-resistant K562/A02 cells. It may serve as an alternative to the current treatment for treating patients with MDR1-overexpressing tumors. Further work is required to validate this drug, and to investigate whether GMZ-1 inhibits the functions of other ATP binding cassette transporters.

\section{Acknowledgements}

The present study was financially supported by the National Natural Science Foundation of China (grant nos. 30901985 and 81600051), the Tianjin Application Foundation and Advanced Technology Research Project (grant no. 15JCQNJC13500), and the Open Fund of Logistics University Central Laboratory of Chinese People's Armed Police Forces (grant no. 2015ZXKF07).

\section{References}

1. Yang CZ, Luan FJ, Xiong DS, Liu BR, Xu YF and Gu KS: Multidrug resistance in leukemic cell line K562/A02 induced by doxorubicin. Zhongguo Yao Li Xue Bao 16: 333-337, 1995.

2. Hackl H, Astanina K and Wieser R: Molecular and genetic alterations associated with therapy resistance and relapse of acute myeloid leukemia. J Hematol Oncol 10: 51, 2017.

3. Dalton WS: Mechanisms of drug resistance in hematologic malignancies. Semin Hematol 34 (4 Suppl 5): S3-S8, 1997.

4. Young AM, Allen CE and Audus KL: Efflux transporters of the human placenta. Adv Drug Deliv Rev 55: 125-132, 2003. 
5. Lee SY, Rhee YH, Jeong SJ, Lee HJ, Lee HJ, Jung MH, Kim SH, Lee EO, Ahn KS, Ahn KS and Kim SH: Hydrocinchonine, cinchonine, and quinidine potentiate paclitaxel-induced cytotoxicity and apoptosis via multidrug resistance reversal in MES-SA/DX5 uterine sarcoma cells. Environ Toxicol 26: 424-431, 2011

6. Dönmez Y, Akhmetova L, Iseri ÖD, Kars MD and Gündüz U: Effect of MDR modulators verapamil and promethazine on gene expression levels of MDR1 and MRP1 in doxorubicin-resistant MCF-7 cells. Cancer Chemother Pharmacol 67: 823-828, 2011.

7. Wibowo M, Levrier C, Sadowski MC, Nelson CC, Wang Q, Holst J, Healy PC, Hofmann A and Davis RA: Bioactive dihydro- $\beta$-agarofuran sesquiterpenoids from the Australian rainforest plant Maytenus bilocularis. J Nat Prod 79: 1445-1453, 2016.

8. Pajeva I and Wiese M: Molecular modeling of phenothiazines and related drugs as multidrug resistance modifiers: A comparative molecular field analysis study. J Med Chem 41: 1815-1826, 1998.

9. Schondörf T, Kurbacher CM, Göhring UJ, Benz C, Becker M, Sartorius J, Kolhagen H, Mallman P and Neumann R: Induction of MDR1-gene expression by antineoplastic agents in ovarian cancer cell lines. Anticancer Res 22: 2199-2203, 2002.

10. Gottesman MM: Mechanisms of cancer drug resistance. Annu Rev Med 53: 615-627, 2002.

11. Anuchapreeda S, Leechanachai P, Smith MM, Ambudkar SV and Limtrakul PN: Modulation of P-glycoprotein expression and function by curcumin in multidrug-resistant human KB cells. Biochem Pharmacol 64: 573-582, 2002.

12. Yu ST, Chen TM, Tseng SY and Chen YH: Tryptanthrin inhibits MDR1 and reverses doxorubicin resistance in breast cancer cells. Biochem Biophys Res Commun 358: 79-84, 2007.
13. Mutoh K, Tsukahara S, Mitsuhashi J, Katayama K and Sugimoto Y: Estrogen-mediated post transcriptional down-regulation of P-glycoprotein in MDR1-transduced human breast cancer cells. Cancer Sci 97: 1198-1204, 2006.

14. Zhou YG, Li KY and Li HD: Effect of the novel antipsychotic drug perospirone on P-glycoprotein function and expression in Caco-2 cells. Eur J Clin Pharmacol 64: 697-703, 2008.

15. Baldwin EL and Osheroff N: Etoposide, topoisomerase II and cancer. Curr Med Chem Anticancer Agents 5: 363-372, 2005.

16. Hande KR: Etoposide: Four decades of development of a topoisomerase II inhibitor. Eur J Cancer 34: 1514-1521, 1998.

17. Kobayashi K and Ratain MJ: Pharmacodynamics and long-term toxicity of etoposide. Cancer Chemother Pharmacol 34 (Suppl): S64-S68, 1994.

18. Smith MA, Rubinstein L, Anderson JR, Arthur D, Catalano PJ, Freidlin B, Heyn R, Khayat A, Krailo M, Land VJ, et al: Secondary leukemia or myelodysplastic syndrome after treatment with epipodophyllotoxins. J Clin Oncol 17: 569-577, 1999.

19. Choudhury RC, Palo AK and Sahu P: Cytogenetic risk assessment of etoposide from mouse bone marrow. J Appl Toxicol 24 115-122, 2004

20. Attia SM, Al-Anteet AA, Al-Rasheed NM, Alhaider AA and Al-Harbi MM: Protection of mouse bone marrow from etoposide-induced genomic damage by dexrazoxane. Cancer Chemother Pharmacol 64: 837-845, 2009.

21. Kapiszewska M, Cierniak A, Papiez MA, Pietrzycka A, Stepniewski M and Lomnicki A: Prolonged quercetin administration diminishes the etoposide-induced DNA damage in bone marrow cells of rats. Drug Chem Toxicol 30: 67-81, 2007.

22. Enomoto R, Koshiba C, Suzuki C and Lee E: Wogonin potentiates the antitumor action of etoposide and ameliorates its adverse effects. Cancer Chemother Pharmacol 67: 1063-1072, 2011. 